\title{
AMC's angular stability improvement through the introduction of lumped components
}

\author{
H. Fernández Álvarez, M.E. de Cos, Member, IEEE, and F. Las-Heras, Senior Member, IEEE
}

\begin{abstract}
A novel method to improve the angular stability of artificial magnetic conductors (AMCs) is presented. The study is carried out from the viewpoint of designers facing common design specifications (resonance frequency, bandwidth and commercially available dielectric). AMCs resonating at $5.8 \mathrm{GHz}$ are designed based on square loop and patch shaped unit cells, introducing lumped components on a later stage aiming to improve the angular stability. Both an equivalent circuit model and electromagnetic simulations are used to provide a clear justification of the presented results. Moreover, some guidelines to choose the proper lumped components' values, using analytical expressions and an AMC's equivalent circuit model, are provided.
\end{abstract}

Index Terms-Artificial Magnetic Conductor (AMC), angular stability, lumped components, reflection coefficient, metasurface.

\section{INTRODUCTION}

$\mathrm{M}$ ETASURFACES are two-dimensional metamaterials, which exhibit unique properties to tailor the propagation of electromagnetic waves. They have proved their usefulness as invisibility cloaks [1], detectors and sensors and absorbers [2-3], among other applications. Artificial Magnetic Conductors (AMCs) are a subset of metasurfaces exhibiting in-phase reflection when a plane wave impinges on them. AMCs are commonly designed as a Frequency Selective Surface (FSS) on a grounded dielectric substrate. They are characterized by computing their reflection coefficient, in both magnitude and phase (the latter varies between $\pm 180^{\circ}$ ). Its operating bandwidth is defined as the range of frequencies with a reflection coefficient phase within $\pm 90^{\circ}$ and a magnitude close to 1 .

There is a wide range of applications for AMCs: as a ground plane for antennas to improve their radiation parameters (BW, efficiency and gain) [4-5], in Radio Frequency Identification (RFID) [6], to reduce the Radar Cross Section (RCS) of certain targets [7-8] and the Specific Absorption Rate (SAR) [9].

Most studies involving AMCs, are based on improving their bandwidth using multi-resonant structures, either by stacking several layers [10] or by using two AMC's with close resonance frequencies [11]. On the other hand, the

This work was supported by the Gobierno del Principado de Asturias/FEDER under Project GRUPIN14-114 and by the Ministerio de Economía y Competitividad under Project TEC2014-54005-P (MIRIIEM).

The authors are with the Electrical Engineering Department, University of Oviedo, 33203 Gijón, Spain (e-mail: hfernandez@tsc.uniovi.es). introduction of lumped inductors to miniaturize them [12] and/or increase their bandwidth [13] has also been presented in the literature. However, only a few contributions deal with improving the AMC's angular stability and most of them are based on multi-layered [14] or complex [15] unit cells. Although both polarizations, Transverse Electric (TE) (electric field normal to the incident plane) and Transverse Magnetic (TM) (magnetic field normal to the incident plane), are usually studied, only a zero degrees' polarization angle is considered.

Consequently, this contribution aims to develop a new technique for improving the angular stability of loop and patch based AMCs, resonating at $5.8 \mathrm{GHz}$, with a proper bandwidth and using a commercially available dielectric. It is known that once the dielectric characteristics of the AMC are fixed, the AMC's geometry and periodicity are the only parameters that can modify its bandwidth and angular stability. RO4003C dielectric $\left(\varepsilon_{r}=3.38\right)$ with a $1.524 \mathrm{~mm}$ thickness $(h)$ is considered. In this work, the AMC's geometrical dimensions are adjusted to achieve a $5.8 \mathrm{GHz}$ resonance frequency.

Moreover, the pros and cons of applying the proposed technique to the loop and patch based AMCs are shown and some design guidelines are provided. HFSS electromagnetic software with proper Master/Slave boundary conditions and a Floquet port excitation are used in the simulations.

\section{ANGULAR STABILITY RESULTS FOR LOOP AND PATCH BASED AMCS}

In this section the angular stability of both loop and patch based AMCs will be analyzed. This analysis will be carried out as follows: the polarization angle of the incident wave $(\phi)$ is varied from $0^{\circ}$ to $90^{\circ}$ in steps of $15^{\circ}$ and for each $\phi$ its incidence angle $(\theta)$ is varied from $0^{\circ}$ to $60^{\circ}$ also in steps of $15^{\circ}$ (see Fig 1(a)). The frequency deviation $\left(\Delta_{f}\right)$ defined as the maximum deviation of the resonance frequency $\left(f_{r}\right)$ and the stable bandwidth $\left(B_{S}\right)$, for which the structure behaves as an AMC no matter the $\theta$, will be computed for each $\phi$. The worst cases (maximum $\Delta_{f}$ and narrowest $B_{s}$ for whichever $\phi$ and $\theta$ ) considering both TE and TM polarization will be shown [16].

\section{A. Loop based AMC}

A square Loop Based AMC (LBAMC) is initially designed to resonate at $5.8 \mathrm{GHz}$ (Fig 1(b)), obtaining the following dimensions: $w_{e}=8.2 \mathrm{~mm}, w_{i}=5.3 \mathrm{~mm}$ and $p=9.2 \mathrm{~mm}$.

AMCs are usually modeled as an equivalent surface impedance $\left(Z_{s}\right)$ which is the parallel connection of the grid and the grounded dielectric impedances [16]. The equivalent 
circuit model of the LBAMC is shown in Fig 1(d). Fig 1(e) depicts the grid inductance's model $\left(L_{g}\right)$ which is due to the metallic strips of the unit cell. The grid capacitance $\left(C_{g}\right)$ is caused by the gap between unit cells and the grounded dielectric slab can be modeled as an inductor $\left(L_{d}\right)$, provided electrically thin dielectrics are considered [16].

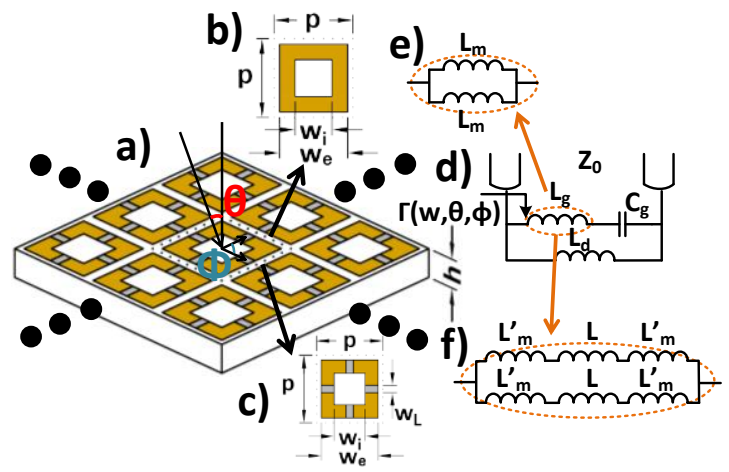

Fig 1. a) Array of several loop based unit cells with inductors, b) geometry of loop based unit cell without (b) and with (c) lumped inductors. (d) Equivalent circuit model of a loop based AMC, e) inductance model for the LBAMC and f) inductance model for the LBAMCI.

The frequency deviation and the stable bandwidth are obtained using HFSS (first row of Table I). As it was analytically study in [17] a Self-Resonant Grid above a grounded dielectric slab is the optimum configuration that analytically avoids the angular dependence of the AMC's resonance frequency ((21) in [17]). However, as it was shown by simulation results in the same paper, there is still certain angular dependence concerning the frequency deviation. From [16] can be concluded that by using highly inductive grids, the AMC's angular stability can be improved. Therefore, lumped inductors are introduced in the middle of each unit cell's side Fig 1(c) (the AMC with these unit cells will be known as Loop Based AMC with lumped Inductors (LBAMCI)). As introducing lumped inductors implies increasing the inductive behavior of the unit cell and hence a reduction of the resonance frequency, the unit cell's dimensions must be reduced to keep the resonance frequency at $5.8 \mathrm{GHz}$. Therefore, the LBAMCI geometric parameters will be: $w_{e}=6.3 \mathrm{~mm}, w_{i}=3.4 \mathrm{~mm}, p=7.3 \mathrm{~mm}$ and $w_{L}=0.8 \mathrm{~mm}$. The width of the unit cell's strips has not been modified since it was wisely chosen to fit 0805 sized inductors [18]. The required lumped inductors' value to get the intended resonance frequency can be estimated through the LBAMCI's equivalent circuit model of Fig 1(d), (f). The value of the grid capacitance $\left(C_{g}\right)$ can be assessed using the formulation presented in [19] for a grid of conductive strips, obtaining $C_{g}=0.203 \mathrm{pF}$. The inductive behavior of the grounded dielectric slab is computed as $L_{d}=\mu h=1.92 n H$ [16]. The grid inductor's value, $L_{g}$, can be estimated from the model of Fig 1(f). The value of $L_{m}^{\prime}$, which models the inductive behavior of the strips, once the inductors are introduced, can be estimated using the formulation proposed by Holloway [20] and thus, a value of $L_{m}^{\prime}=0.805 \mathrm{nH}$ is obtained. Once $C_{g}, L_{m}^{\prime}$ and $L_{d}$ are known, the value of the lumped inductors, $L$, can be solved to get $f_{r}=5.8 \mathrm{GHz}$. Therefore, a value of $L=$
$1.99 \mathrm{nH}$ for the lumped inductors is analytically obtained. Fullwave electromagnetic simulations, by sweeping $\mathrm{L}$ about the aforementioned analytical value, give $L=1.8 \mathrm{nH}$ to get $f_{r}=5.8 \mathrm{GHz}$. This proves the validity of the presented method to estimate the required lumped inductors' value that provides the intended resonance frequency. The error in the inductor's value estimation is contributable to the approximations used in the formulation (the grid inductance is estimated using the inductance of a microstrip line; it is assumed an electrically thin dielectric slab...).

TABLE I

ANGULAR STABILITY OF LOOP BASED AMC

\begin{tabular}{lcccc}
\hline \hline & $\Delta_{f}[\mathrm{MHz}]$ & $\Delta_{f}[\%]$ & $B_{s}[\mathrm{MHz}]$ & $B_{s}[\%]$ \\
\hline LBAMC & 384.1 & 6.43 & 183 & 3.04 \\
LBAMCI & 186 & 3.17 & 220 & 3.82 \\
\hline \hline
\end{tabular}
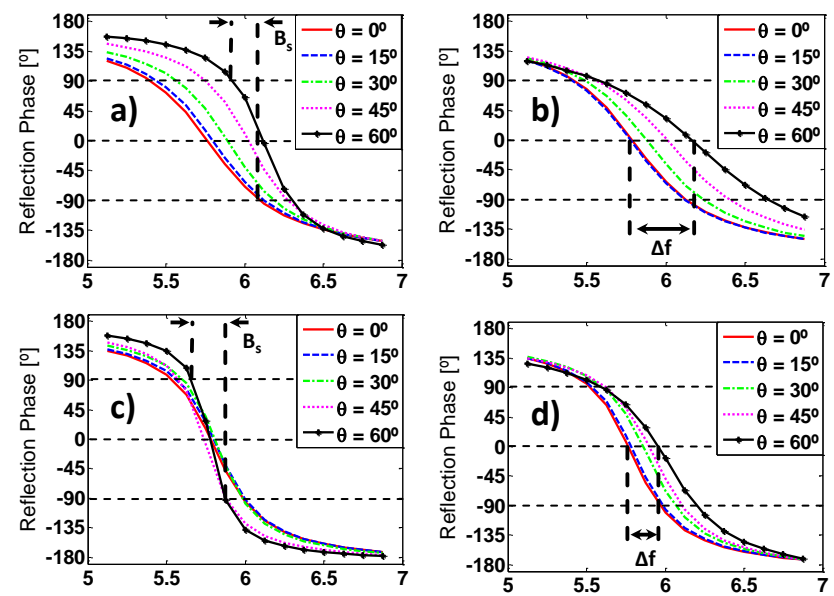

Fig 2. Worst case angular stability results of LBAMC ( $\phi=90^{\circ}$ TE (a) and $\left.\phi=0^{\circ} \mathrm{TM}(\mathrm{b})\right)$ and LBAMCI ( $\phi=0^{\circ} \mathrm{o}$ TE (c) and $\phi=0$ o TM (d)).

The angular stability analysis of the LBAMCI is presented in the second row of Table I. By comparing the results of LBAMC and LBAMCI in terms of $\Delta_{f}$ and $B_{s}$, it can be concluded that the introduction of lumped inductors in the unit cells improves both, the frequency deviation and the stable bandwidth. Moreover, a miniaturization of the AMC's unit cell is also achieved, allowing to arrange more unit cells in the same space and hence approaching in a greater extent to the infinite behavior of the simulated AMC. The worst case angular stability results of LBAMC and LBAMCI are shown graphically in Fig 2 to further clarify the analysis carried out.

\section{B. Patch based AMC}

The angular stability of a square Patch Based AMC (PBAMC) is now analyzed. Fig 3(b) depicts the AMC's unit cell. To reach the targeted resonance frequency $(5.8 \mathrm{GHz})$, the unit cell's geometric parameters must be: $p=11 \mathrm{~mm}$ and $w_{e}=10 \mathrm{~mm}$.

The PBAMC's equivalent circuit model is shown in Fig 3(d). Fig 3(e) shows the grid capacitance's model which arise from the gap between neighbor unit cells. As it was said, the grounded dielectric can be modeled as an inductor $\left(L_{d}\right)$.

The first row of Table II shows worst case angular stability results of this PBAMC. Lumped capacitors are introduced 
between neighboring unit cells (Fig 3(c)), to improve the angular stability of patch based AMCs. As with the LBAMCI, the unit cell periodicity should be reduced to achieve $f_{r}=$ $5.8 \mathrm{GHz}$ once the lumped capacitors are introduced. Thus, the geometric parameters of this Patch Based AMC with lumped Capacitors (PBAMCC) are: $p=8.9 \mathrm{~mm}, w_{e}=7.9 \mathrm{~mm}$ and $w_{C}=1.26 \mathrm{~mm}$. The value of the lumped capacitors can be estimated using the equivalent circuit model of the PBAMCC (Fig 3(d)) and the grid capacitance's model (Fig 3(f)). The inductor due to the grounded dielectric has the same value as in LBAMCI $\left(L_{d}=1.92 n H\right)$, since the dielectric thickness is not varied. The value of $C_{g}^{\prime}$, which is the grid capacitance between neighboring unit cells once the lumped capacitance is introduced, can be estimated using the edge capacitance used in [21], obtaining $C_{g}^{\prime}=0.135 p F$. Knowing these two values $\left(L_{d}\right.$ and $C_{g}^{\prime}$ ), the value of the lumped capacitor $(C)$ is estimated from the resonance frequency of the AMC's equivalent circuit model, given that $f_{r}=5.8 \mathrm{GHz}$ is sought. Therefore, solving for $C$, a value of $0.12 \mathrm{pF}$ is obtained. Performing electromagnetic simulations of the AMC using lumped capacitor's values about the analytically obtained one, a value of $0.1 \mathrm{pF}$ is needed to get the desired resonance frequency. Thus, the validity of the analytical approximation is verified.

The second row of Table II shows the angular stability results of the PBAMCC. Although it seems from the simulations results that the frequency deviation is slightly reduced, this cannot be confirmed from an analytical point of view. However, what it is possible to ensure is that the stable bandwidth is greatly improved when capacitors are introduced between unit cells.

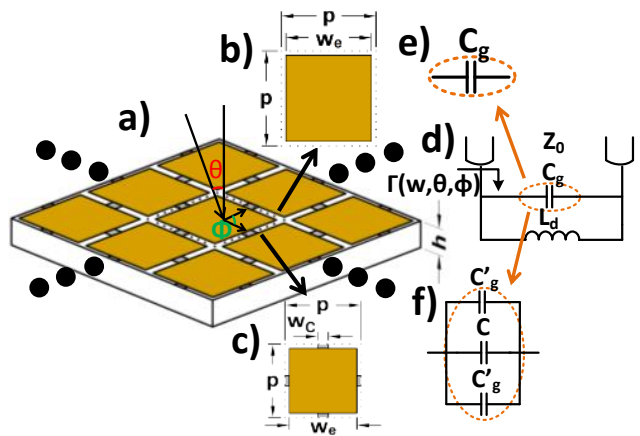

Fig 3. a) Array of several patch based unit cells with capacitors, geometry of patch based unit cell without (b) and with (c) lumped capacitors. d) Equivalent circuit model of a patch based AMC, e) grid capacitance model for the PBAMC and $f$ ) grid capacitance model for the PBAMCC.

TABLE II

ANGULAR STABILITY OF PATCH BASED AMC

\begin{tabular}{lcccc}
\hline & $\Delta_{f}[\mathrm{MHz}]$ & $\Delta_{f}[\%]$ & $B_{s}[\mathrm{MHz}]$ & $B_{s}[\%]$ \\
\hline$P B A M C$ & 655 & 11 & 220 & 3.72 \\
$P B A M C C$ & 618 & 9.9 & 516.1 & 8.62 \\
\hline \hline
\end{tabular}

C. Comparison between loop and patch based AMCs using the previous technique

As it was shown in the previous sections, the angular stability of loop and patch based AMCs can be improved by introducing lumped components (inductors and capacitors, respectively). The LBAMC and LBAMCI provide a smaller frequency deviation than both PBAMC and PBAMCC, respectively. However, the stable bandwidth is wider if PBAMC and PBAMCC are considered. The latter is even more evident when lumped capacitors are introduced (PBAMCC). Thus, the choice of loop or patch based AMC relies entirely on the intended application and its bandwidth/stability requirements. On the other hand, when the AMC has to face small area constraints, the LBAMCI is the best option due to the higher miniaturization rendered by it.

\section{Method to improve angular stability.}

Once the previous structures are analyzed, a method to improve the angular stability of loop and patch based AMCs can be provided.

1. According to the requirements of the intended application, choose either a loop or patch shaped AMC.

2. Design the AMC taking into consideration the lumped component sizes (that will be introduced) and the fabrication tolerances and restrictions.

3. Estimate the required lumped components value (inductors or capacitors).

4. Use electromagnetic simulations to adjust the proper lumped component value, using the estimated one as starting point and considering the ones commercially available whose self-resonance frequency is well above the AMC's operation band.

5. If the lumped component is not commercially available, you should slightly readjust the unit cell geometrical dimensions up to an available component can be used.

\section{E. Explanation of the phenomenon using a modelling.}

Following the work presented in [16], the analytical expressions that can be used for modelling the grounded dielectric slab $\left(Z_{d}\right)$ and the grid impedance $\left(Z_{g}\right)$ of both patch and loop based AMCs for TE and TM polarizations are:

$$
\begin{gathered}
Z_{d}^{T E} \approx j w L_{d} \\
Z_{d}^{T M} \approx j w L_{d} \cos ^{2}\left(\theta_{2}\right) \\
\theta_{2}=\arcsin \left(\frac{\sin (\theta)}{\sqrt{\varepsilon_{r}}}\right) \\
Z_{g_{\text {patch }}}^{T E}=\left(j w C_{g}\left(1-\frac{\sin ^{2}(\theta)}{\varepsilon_{r}+1}\right)\right)^{-1} \\
Z_{g_{\text {loop }}}^{T E}=\left(j w L_{g}+\left(j w C_{g}\right)^{-1}\right)\left(1-\frac{\sin ^{2}(\theta)}{\varepsilon_{r}+1}\right)^{-1} \\
Z_{g_{\text {patch }}}^{T M}=\left(j w C_{g}\right)^{-1} \\
Z_{g_{\text {loop }}^{T M}}^{T M}=j w L_{g}+\left(j w C_{g}\right)^{-1}
\end{gathered}
$$

For TE, $Z_{d}^{T E}$ (1) does not depend on the incidence angle, but $Z_{g}$ for both patch (4) and loop (5) based AMCs does. However, increasing $C_{g}$ for the patch based AMC brings about a decrease of $Z_{g_{\text {patch }}}^{T E}$ (4) and hence, an incidence angle dependence reduction. On the other hand, for the loop based AMC increasing $L_{g}$ mitigates the variation of $Z_{g_{\text {loop }}}^{T E}$ with the incidence angle [16].

For the TM case, $Z_{d}^{T M}$ depends on the incidence angle. Nevertheless, increasing $C_{g}$ for the patch based AMC, gives rise to a decrease in $Z_{g_{p a t c h}}^{T M}$ and hence, this lower value dominates in the AMC's surface impedance $\left(Z_{s}\right)$ (parallel connection between (6) and (2)). On the other hand, increasing 
$L_{g}$ in the loop based AMC compensates the variation of $Z_{d}^{T M}$ with the incidence angle.

As it was previously shown the reduction of the $Z_{s}$ angular dependence improves both $\Delta_{f}$ and $B_{s}$ for the LBAMCI and maintains $\Delta_{f}$ and widens $B_{s}$ for PBAMCC with regards to LBAMC and PBAMC, respectively.

\section{CONCLUSION}

A new technique to improve the angular stability of loop and patch based AMCs through the introduction of lumped components (inductors and capacitors, respectively) has been presented. An analytical study of both AMCs to get a proper estimation of the inductor's and capacitor's values required to achieve a $5.8 \mathrm{GHz}$ resonance frequency has been shown. As a reward for the introduction of lumped components not only an enhancement of the angular stability has been proved but also the possibility of miniaturizing the whole AMC. Although a square shaped loop and a patch based AMC have been considered in this paper, this technique can be spread to other AMC's unit cell geometries as well.

The method described in this paper overcomes previously different approach (already in the literature) [14,16-17] in terms of angular stability improvement. On the other hand, the technique introduced in [22] gives rise to a multilayer structure and even though it achieves a small frequency deviation, a uniaxial complex structure design is needed. In addition, none of the mentioned contributions carry out a complete angular stability analysis.

Increasing structural capacitance or inductance can give rise to similar behavior but this is more difficult to predict. Changing the structural elements not only do the inductance and/or the capacitance behavior of the AMC change, but also its capacitance and/or inductance behavior respectively. So, it is not easier to increase the inductance (capacitance) behavior without modifying considerably the capacitance (inductance) one. Therefore, as a general conclusion regarding AMC angular stability, it can be said that the introduction of lumped components outperforms the design of structures that achieve the same inductive or capacitive behavior through structural features. This is not only because they are easier to be designed, but also because their design parameters and their gap between neighbor unit cells can be controlled (which is crucial in the AMC angular stability behavior [16]).

Furthermore, the limitation in the angular margin that can be measured using a quasi-monostatic configuration set-up [23], gives rise to use indirect methods to both test an AMC's performance and prove its utility. An example of such an indirect method could be the combination of AMCs with other electromagnetic devices, such as antennas or waveguides, to test the latter improved performance due to the AMC's presence. Indeed, authors are currently working in the miniaturization and radiation properties enhancement of an antenna operating at frequencies below $1 \mathrm{GHz}$, by applying this methodology.

\section{REFERENCES}

[1] P.Y. Chen and A. Alu, "Mantle cloaking using thin patterned metasurfaces," Phys. Rev. B, vol. 84, no 20, p. 205110, 2011.

[2] H. F. Álvarez, M.E. de Cos and F. Las-Heras, "A Six-Fold Symmetric Metamaterial Absorber," Materials. vol. 8, no. 4, pp. 1590-1603, 2015.

[3] H.F. Álvarez, M.E. de Cos and F. Las-Heras, "A Thin C-Band Polarization and Incidence Angle-Insensitive Metamaterial Perfect Absorber," Materials, vol. 8, no. 4, pp. 1666-1681, 2015.

[4] A. P. Feresidis, G. Goussetis, Shenhong Wang and J. C. Vardaxoglou, "Artificial magnetic conductor surfaces and their application to lowprofile high-gain planar antennas," in IEEE Trans. Antennas Propag., vol. 53, no. 1, pp. 209-215, 2005.

[5] N. Engheta and R.W. Ziolkowski, "Metamaterials: physics and engineering explorations," John Wiley \& Sons, 2006.

[6] R. C. Hadarig, M. E. D. Cos, Y. A. Lopez and F. Las-Heras, "Novel bow-tie antenna on artificial magnetic conductor for $5.8 \mathrm{GHz}$ radio frequency identification tags usable with metallic objects," in IET Microwaves Antennas Propag., vol. 5, no. 9, pp. 1097-1102, 2011.

[7] J. C. I. Galarregui, A. T. Pereda, J. L. M. D. Falcon, I. Ederra, R. Gonzalo, and P. D. Maagt, "Broadband radar cross-section reduction using AMC technology," IEEE Trans. Antennas Propag., vol. 61, no. 12, pp. 6136-6143, 2013.

[8] M. E. de Cos, Y. Alvarez-Lopez, and F. Las Heras Andres, "On the influence of coupling AMC resonances for RCS reduction in the SHF band," Prog. Electromagn. Res., vol. 117, 103-119, 2011.

[9] M. Mantash, M. E. de Cos, A. C. Tarot, S. Collardey, K. Mahdjoubi and F. Las-Heras, "Dual-band textile hexagonal artificial magnetic conductor for WiFi wearable applications," European Conference on Antennas and Propagation (EUCAP), Prague, 2012, pp. 1395-1398.

[10] Y. Ranga, L. Matekovits, A. R. Weily, and K. P. Esselle, "A Constant Gain Ultra-Wideband Antenna With A Multi-Layer Frequency Selective Surface," Prog. Electromagn. Res., vol. 38, pp. 119-125, 2013.

[11] M. E. de Cos, Y. Alvarez Lopez, and F. Las-Heras, "A novel approach for RCS reduction using a combination of artificial magnetic conductors," Prog. Electromagn. Res., vol. 107, pp. 147-159, 2010.

[12] R. Saad and K. L. Ford, "A miniaturised dual band Artificial Magnetic Conductor using lumped components," Loughborough Antennas \& Propagation Conference, Loughborough, 2011, pp. 1-4.

[13] M. G. Bray and D. H. Werner, "Fast simulation of lumped-element loaded AMC antenna systems using embedded element theory," 2010 IEEE Antennas and Propagation Society International Symposium, Toronto, 2010, pp. 1-4.

[14] M. Hosseini, A. Pirhadi, and M. Hakkak, "A Novel AMC With Little Sensitivity To The Angle Of Incidence Using 2-Layer Jerusalem Cross FSS," Prog. Electromagn. Res., vol. 64, pp. 43-51, 2006.

[15] A. Monorchio, G. Manara, and L. Lanuzza, "Synthesis of artificial magnetic conductors by using multilayered frequency selective surfaces," IEEE Antennas Wirel. Propag. Lett., vol. 1, no. 1, pp. 196199, 2002.

[16] M. E. de Cos and F. Las-Heras, "On the advantages of loop-based unitcell's metallization regarding the angular stability of artificial magnetic conductors," J. Appl. Phys. A, vol. 118, no. 2, pp. 699-708, 2014.

[17] C. Simovski, P. de Maagt, I. Melchakova, "High-impedance surfaces having stable resonance with respect to polarization and incidence angle", IEEE Trans. Antennas Propag., vol. 53, no. 3, pp. 908-914, 2005.

[18] Chip Inductors - 0805HT. (n.d.). Coilcraft. [Online]. Available: http://www.coilcraft.com/pdfs/0805ht.pdf. Accessed Ap. 4, 2017.

[19] S. Tretyakov, Analytical modeling in applied electromagnetics. Boston: Artech House, 2003.

[20] C. L. Holloway and E. F. Kuester, "Net and partial inductance of a microstrip ground plane," IEEE Trans. Electromagn. Compat., vol. 40, no. 1, pp. 33-46, 1998.

[21] D. F. Sievenpiper, "High-Impedance Electromagnetic Surfaces," Ph.D. dissertation, Electrical Engineering, University of California, Los Angeles, California, 1999.

[22] S. Hashemi, S. Tretyakov, M. Soleimani, C. Simovski, "Dual-polarized angularly stable high-impedance surface", IEEE Trans. Antennas Propag., vol. 61, no. 8, pp. 4101-4108, 2013.

[23] H. Fernandez Alvarez, M. E. de Cos Gomez and F. Las-Heras, "Angular Stability of Metasurfaces: Challenges Regarding Reflectivity Measurements [Measurements Corner]," in IEEE Antennas and Propag. Mag., vol. 58, no. 5, pp. 74-81, Oct. 2016. 